\title{
CORRIGENDUM
}

\section{Wind-tunnel tests of a heavy-class helicopter optimised for drag reduction - CORRIGENDUM}

\author{
A. Zanotti, G. Droandi, G. Gibertini, D. Grassi, G. Campanardi, F. Auteri, A. Aceti \\ and A. Le Pape
}

DOI: 10.1017/aer.2016.60, Published online: 3 June 2016

The authors were listed incorrectly in the article by Zanotti ${ }^{(1)}$. The authors should have appeared in the following order:

G. Gibertini, A. Zanotti, G. Droandi, D. Grassi, G. Campanardi, F. Auteri, A. Aceti and A. Le Pape

The article has now been updated with all authors listed in the correct order. This change has been made to the online and print PDF and HTML copies.

\section{REFERENCE}

1. Zanotti, A., Droandi, G., Gibertini, G., Grassi, D., Campanardi, G., Auteri, F., Aceti, A. and Le PAPE, A. Wind-tunnel tests of a heavy-class helicopter optimised for drag reduction, Aeronautical $J$, DOI: 10.1017/aer.2016.60, first published online 3 June 2016. 